\title{
A molecular jack-of-all-trades
}

\author{
DNA is much more than the genetic information it carries. It is a versatile material for creating systems with \\ tailor-made functionalities that are having an important impact in emerging technologies.
}

N ucleic acids and associated proteins are essential molecular curators that store, preserve, translate and duplicate the genetic information of living organisms. Having understood how proteins are encoded and translated from genetic sequences of DNA, researchers quickly realized the potential of engineering such processes for alternative purposes. Currently, gene editing and DNA reactions are deeply integrated into research and technological environments, and even in our daily life. For instance, the gold standard diagnostic test for severe acute respiratory syndrome coronavirus 2 (SARS-CoV-2) infection is the real-time reverse-transcription polymerase chain reaction (RT-PCR) test; which is nothing more than a lab-based adaptation of enzymatic reactions with nucleic acids that occur naturally in cells for viral RNA detection.

Beyond these more traditional roles, DNA and its biochemistry are also established tools for the design and fabrication of functional materials for various applications $s^{1,2}$. The specificity of Watson-Crick base-pairing and our ability to define DNA sequences results in an endless supply of programmable interactions. These interactions enable the rational design and fabrication of one-dimensional (1D), 2D and 3D materials with distinct structural, mechanical and functional properties. DNA materials consisting of short strands, ordered nanostructures, multicomponent assemblies or bulk hydrogels have been fabricated for applications in bioimaging, catalysis, plasmonics, molecular computing and drug delivery ${ }^{1-4}$. To further highlight the versatility of DNA as a materials engineering tool, in this issue of Nature Materials we present several research articles on DNA materials with applications in virus binding and inhibition, as well as data storage.

DNA programmability and specificity can be harnessed to create structural DNA building blocks that self-assemble into intricate, multicomponent nanostructures ${ }^{1,5}$. Nonetheless, predicting the performance of such constructs and encoding further structural complexity is challenging as most DNA design software only considers the geometrical layout or has limited structural diversity. In an Article in this issue, Hai-Jun $\mathrm{Su}$, Carlos Castro and colleagues present

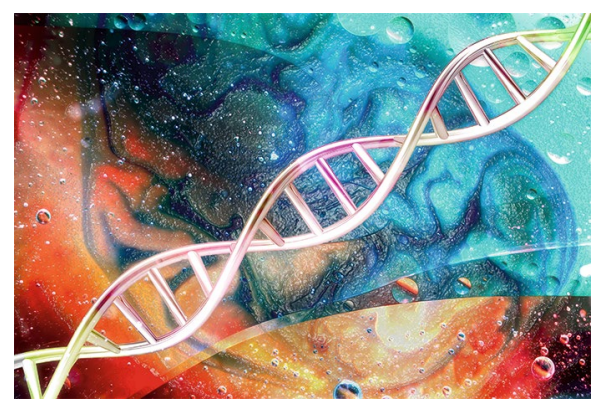

Credit: Olivier Verriest / Alamy Stock Photo

the software MagicDNA for the integrated computational engineering of DNA origami with high structural and dynamic complexity. It combines computer-aided design and engineering based on coarse-grained molecular dynamics to allow for intricate design at multiple scales. Thus, precise control of the static and dynamic properties of the DNA origami is achieved via fast automation. The authors showcase the advantages of the software by designing and then fabricating a series of large, multicomponent and reconfigurable DNA structures.

The inherent biocompatibility of DNA materials makes them particularly suitable for biomedical applications, which has witnessed the development of DNA nanodevices for drug delivery, vaccines and viral inhibition ${ }^{2,6,7}$. An Article by Hendrik Dietz and colleagues describes the design and fabrication of programmable triangular DNA origami building blocks that self-assemble into icosahedral shells of distinct size and complexity. Realizing the potential of these DNA hollow shells for physical trapping, the authors designed half-shell icosahedral structures with apertures large enough to trap virus particles inside the shells. By functionalizing these DNA structures with antiviral antibodies, the authors successfully fabricated virus-binding and inhibiting agents that decrease viral infection and cellular viral loads. As noted by Neha Chauhan and Xing Wang in a related News \& Views article, not only do these shells hold promise for the development of clinical therapeutics against viral infections, but they can also be easily adapted into virus-like particles for vaccination or drug delivery by functionalization of the external surface with antigens.
The biological information storage capabilities of DNA are also being explored to create alternative memory materials for durable and high-density digital data-storage. Typically, in DNA data storage, information is encoded into nucleotide sequences that are stored in a support material to protect this information and allow for data access ${ }^{8}$. A crucial feature of any archival system is the ability to efficiently and reliably access specific information. Mark Bathe and colleagues describe in an Article a PCR-free random-access system for DNA data storage. DNA containing particular information is physically encapsulated in silica beads whose surface is labelled with multifunctional DNA barcodes. These DNA strands act as data identifiers and also enable for file and dataset selection based on Boolean logic. Search results can then be physically retrieved using fluorescent sorting. Because this approach doesn't require the amplification steps associated with traditional PCR-based random-access systems, it is not affected by the non-specific crosstalk between file sequences and barcodes and simplifies the search process in a DNA data pool. In an accompanying News \& Views article, Luca Piantanida and William Hughes discuss how these features, in particular the ability to use conditional logic to sort data files made from DNA, are an important step forward in translating DNA data storage into real-life devices.

DNA is certainly a remarkable molecule with vast applications beyond its main biological role. Supported by these recent examples, it is not unrealistic to envision technologies based on DNA material properties. Moreover, it makes one wonder what else this life-giving material can achieve.

Published online: 25 August 2021 https://doi.org/10.1038/s41563-021-01096-y

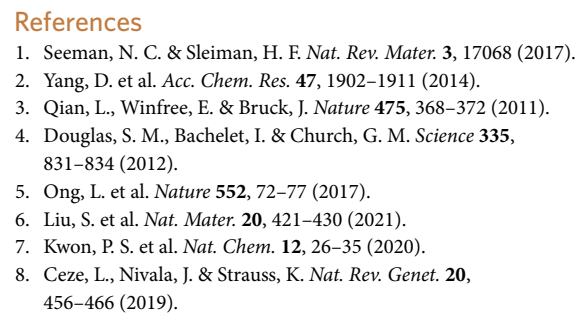

